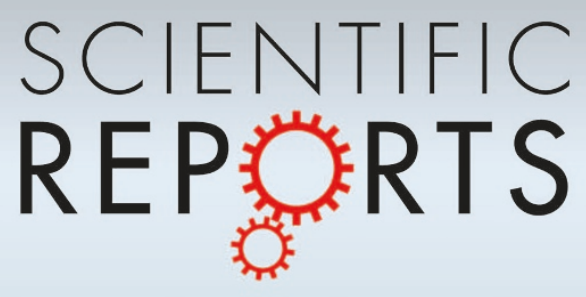

OPEN

SUBJECT AREAS:

NANOPARTICLES

CARBON NANOTUBES AND

FULLERENES

Received

28 November 2013

Accepted

21 May 2014

Published

10 June 2014

Correspondence and requests for materials should be addressed to

J.-C.V. (valmalette@ univ-tln.fr)

\section{Raman scattering of linear chains of strongly coupled Ag nanoparticles on SWCNTs}

\author{
Jean-Christophe Valmalette ${ }^{1,2}$, Zhenquan Tan ${ }^{3}$, Hiroya Abe ${ }^{3} \&$ Satoshi Ohara $^{3}$
}

\author{
'Université de Toulon, BP 20132, F-83957 La Garde Cedex, France, ${ }^{2}$ CNRS, IM2NP (UMR 7334), BP 20132, F-83957 La Garde \\ Cedex, France, ${ }^{3}$ Osaka University, Joining \& Welding Research Institute, Ibaraki, Osaka 5670047, Japan.
}

We compare the Raman scattering properties of hybrid nanostructures consisting of Ag nanoparticles (NPs) in disordered and aligned arrangements on single-walled carbon nanotubes (SWCNTs) as a result of chemical and photoreduction methods. In the latter case, the unique structure of the very small Ag NP (from 4 to $7 \mathrm{~nm}$ ) chains generated an extremely large mode at $969 \mathrm{~cm}^{-1}$ that was assigned to the sulphate-silver interaction at the NP surface. Another strong mode was present at $1201 \mathrm{~cm}^{-1}$ and was assigned to an IR-active mode of sodium dodecyl sulphate (SDS); this mode was observed because the symmetry changes altered the selection rules. We demonstrate that both the UV photoreduction of silver and the presence of SWCNTs are necessary to produce this very strong Raman scattering. The Raman modes of the SWCNTs are also significantly modified by the presence of Ag NP chains along the nanotubes.

F unctionalisation of carbon nanotubes (CNTs) by noble metal nanoparticles (NPs) has been recently studied for applications in nanotechnology, including sensors ${ }^{1,2}$, molecular electronics, photonics, energy conver$\operatorname{sion}^{3}$ and storage $e^{4}$, light emission and bio-labelling ${ }^{5,6}$. The unusual properties that result from the combination of the unique structure of the CNTs and the enhanced electromagnetic field (EF) induced by the interaction between the noble metal NPs and light are beneficial for label-free detection. Recently, it has been shown that discrete particles could be more suitable than rough films for detecting molecules ${ }^{3}$. The predicted strong EF enhancement induced by Ag-decorated CNT nanostructures has been demonstrated very recently in singlemolecule surface-enhanced Raman scattering (SMSERS) experiments on pyridine molecules ${ }^{7}$. However, the development of a well-controlled nanoantenna ${ }^{8-10}$ that combines high resolution, strong EF enhancement and good mechanical properties is one of the main challenges for sub-wavelength label-free spectroscopy. Subwavelength label-free spectroscopy includes tip-enhanced Raman scattering (TERS) ${ }^{11-13}$ and tip-enhanced infrared scattering (TEIRS) ${ }^{14,15}$. CNTs that are functionalised by silver NPs could be used as local probes to simultaneously provide nanoscale resolution for surface imaging and chemical identification by near-field spectroscopy.

Many techniques for the chemical synthesis of silver-decorated CNTs are described in the literature ${ }^{16-18}$. However, theoretical predictions show that the EF strongly depends on how the NPs are organised on the CNTs. For example, dimers and trimers of silver NPs have been demonstrated to produce strong Raman scattering enhancement ${ }^{19}$. Electromagnetic interaction in a $1 \mathrm{D}$ array obtained by electron beam lithography induces a near-field coupling between metal $\mathrm{NPs}^{20,21}$. A theoretical calculation predicted that arrays of carbon nanotubes filled with silver cylinders generate $10^{6}$ enhancement in the Raman signal of absorbed molecules ${ }^{22}$.

Sodium dodecyl sulphate (SDS) molecules can induce a well-organised molecular structure, as shown by Tan et al. ${ }^{23}$, for palladium-decorated single-walled carbon nanotubes (SWCNTs). These authors suggested that the palladium NP helix structure results from the formation of a self-organisation mechanism of the SDS molecules on the surface of the SWCNTs due to the inter-chain van der Waals interaction between the alkyl chains. However, the ionic sulphate group is expected to interact with the silver NP surface, whereas the hydrophobic alkyl chains adsorb on the SWCNTs. The final structure depends on the interactions between the following three pairs: bridging molecules and SWCNTs; bridging molecules and NPs; and the molecules themselves.

The morphologies of Ag@CNT nanostructures have been described by scanning probe and electron microscopy, but, to our knowledge, the chemical and physical interactions among CNTs, bridging molecules and NPs have not been studied due to the very low number of SDS molecules and their relatively low cross-section scattering. In this paper, we investigate the possibility of stabilising very small silver NPs $(<10 \mathrm{~nm})$ onto SWCNTs by using SDS molecules to locally enhance the EF via the combination of two resonant single 
nano-objects (SWCNTs and Ag NPs) to obtain signals from localised interactions. The nanostructures were obtained by both chemical reduction and the photoreduction of silver salt in a suspension of SDS-functionalised SWCNTs. The physical characterisation was performed using transmission electron microscopy, X-ray diffraction, infrared spectroscopy, UV-vis absorption and Raman spectroscopy.

\section{Results}

As shown in Figure 1, the morphology of Ag@SDS@SWCNTs depends strongly on the method used for the silver salt reduction. Photoreduction leads to small homogeneous particles (from 4 to $7 \mathrm{~nm}$ ) that are well aligned on both the isolated SWCNTs and the SWCNT bundles. Several silver particles that are separated from the nanotubes are also visible. These results suggest that the photoreduction method induces a strong interaction between silver particles and carbon nanotubes. In comparison, the chemical reduction of the silver particles onto SWCNTs results in larger particles (from 15 to $25 \mathrm{~nm}$ ) with a higher size dispersion. These findings are consistent with previously reported results on the reduction of noble metal salts $^{24}$. Additionally, the photoreduction method also prevents residual chemical impurities from occurring.

These results show that the silver NPs can be synthesised onto SWCNTs using the supramolecular self-assembly of SDS molecules that are wrapped around the SWCNTs. SDS molecules are adsorbed to the CNT surface through hydrophobic interactions between the hydrophobic linear chains of the SDS molecules and the sidewall of the $\mathrm{CNTs}^{25,26}$ (see Figure 2 and Supplementary Information Figure $\mathrm{S} 1$ ). Both the single nanotubes and the bundles covered by SDS layers are present in the suspension. However, the SDS molecules also form micelles in the suspension around the nanotubes. Only a few studies have been published on the use of SDS as a stabilising agent for silver $\mathrm{NPs}^{27}$. As shown in Figure 1, the size and morphology of the silver NPs strongly depend on the reduction conditions for the silver salt. UV photoreduction results in a homogeneous distribution of silver NPs with sizes ranging from 4 to $7 \mathrm{~nm}$ that are localised along the SWCNT bundles with similar inter-particle distances ranging from 2 to $10 \mathrm{~nm}$. This complex nanostructure is stabilised by the combination of the SDS/SWCNT hydrophobic interactions and the strong interaction between the polar sulphate group of SDS and the silver surface. We observed more isolated NPs in the silver samples than in the Pd@SDS@SWCNTs (see Supplementary Information Figure S2). This result can be explained by the fact that the ionic exchange between $\mathrm{Na}^{+}$and $\mathrm{Ag}^{+}$is less efficient than the exchange between $\mathrm{Na}^{+}$and $\mathrm{Pd}^{2+}$ because of differences in the radius (128 $\mathrm{pm}$ for $\mathrm{Ag}^{+}$ and $73 \mathrm{pm}$ for $\mathrm{Pd}^{2+}$ ) and the ionic charges.

Several characteristic features were observed in the Raman spectra of the SWCNTs, and each of these features can be used for structural and electronic characterisation ${ }^{28,29}$. As shown in Figure 3, the lowfrequency radial breathing modes (RBMs), which are associated with a uniform radial motion of the atoms of the entire tube, confirm that the CNTs are single walled. The frequency of the RBM barely depends on the chiral angle of the tube but is inversely proportional to the diameter according to the empirical relation $d=248 / \omega$, where $\omega$ represents the Raman shift. From our experimental data, we can calculate that the pure SWCNTs used in this study have diameters ranging from 0.95 to $1.35 \mathrm{~nm}$. When SDS was absorbed onto the SWCNTs, both the position and the relative intensities of the RBM
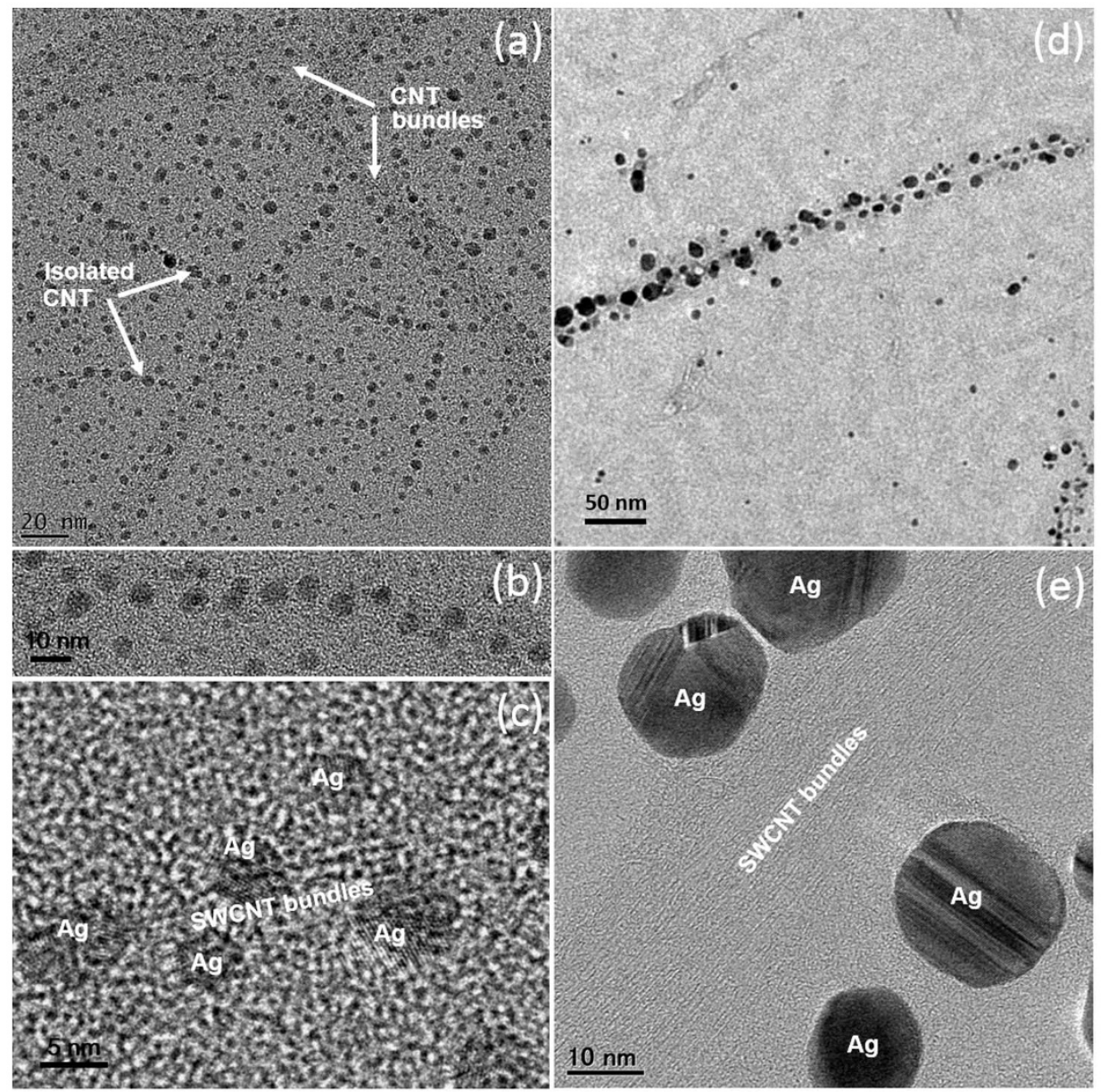

Figure 1 | TEM micrographs of Ag nanoparticles precipitated onto SDS-functionalised SWCNTs by photoreduction (UV-Ag@SDS@SWCNTs, $(\mathrm{a}-\mathrm{c})$ ) and by chemical reduction (Chem-Ag@SDS@SWCNTs, (d-e)). 
(a)

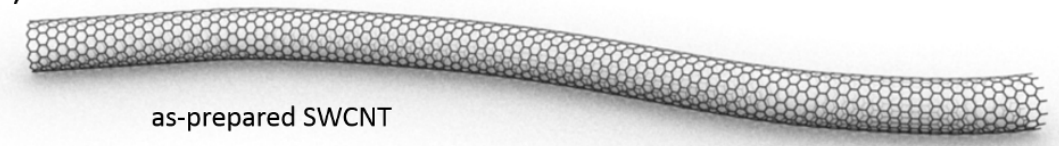

(b)

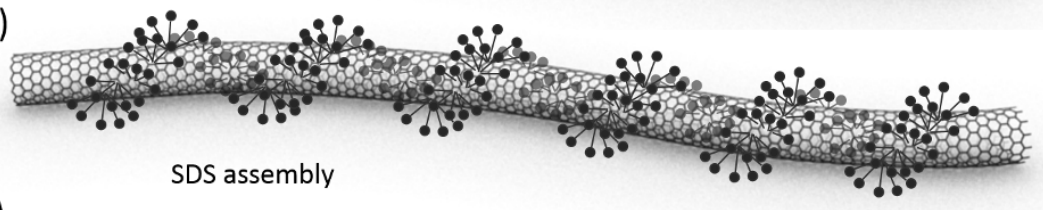

(c)
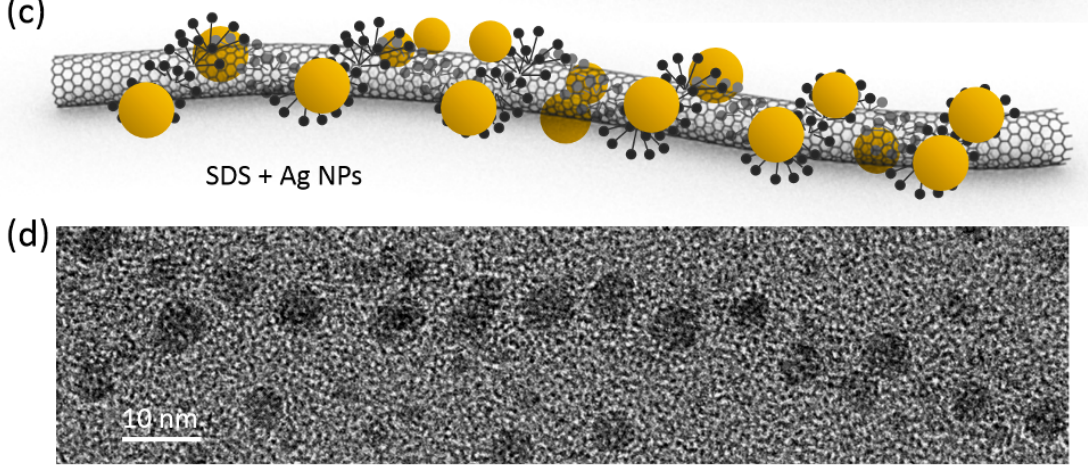

Figure $2 \mid$ Schematic representation for Ag NPs created from a template on SDS-wrapped SWCNT bundles.

were modified (Figure 3b), which may be attributed to the charge transfer that is enhanced by the strong hydrophobic interaction of the SDS molecules with the sidewall $\pi$-electron of the SWCNTs; this enhancement is associated with a significant change in the electronic density of the state of the SWCNTs ${ }^{30}$. The RBM spectral region is strongly modified in the UV-Ag@SDS@SWCNT samples: the single RBM modes are not visible, and a new broad and strong mode appears at $226 \mathrm{~cm}^{-1}$. Conversely, the introduction of palladium (Pd@SDS@SWCNTs, see Supplementary Information Figure S3) or silver by chemical reduction (Chem-Ag@SDS@SWCNTs) does not affect the RBM region as much. However, the intensities of the D-band $\left(1330 \mathrm{~cm}^{-1}\right)$ of both pure SWCNTs and SDS@SWCNTs are relatively weak compared to the G-band $\left(1581 \mathrm{~cm}^{-1}\right)$. These results indicate that SWCNTs have a very high graphitisation purity and that SDS functionalisation does not affect the band intensity significantly. By contrast, the UV-irradiated SDS@SWCNTs show a slight increase in this ratio due to the formation of an irradiation-induced defect. The D-band/G-band intensity ratio is significantly higher for the UV- and Chem-Ag@SDS@SWCNTs (16\% and 11\%, respectively) than for the pure SWCNTs and SDS@SWCNTs $(2.9 \%$ and $3.1 \%$, respectively) (Figure 3c). The maximum of the D-band and the G-band shifted slightly to a high-frequency region. The higher Dband/G-band intensity ratio observed for the UV-Ag@SDS@ SWCNT samples indicates that the silver reduction may have introduced some defects in the SWCNTs. This observation is correlated to a significant increase in the FWHM of the D-band, which is generally used to evaluate the carbon impurity that results from common $\mathrm{sp}^{2}$ hybridised carbon ${ }^{28}$. In addition, we observed a modification of the shape of the $\mathrm{G}^{\prime}$ band at $2648 \mathrm{~cm}^{-1}$ after the SDS functionalisation and the UV irradiation treatment. The intensity of the $2 \mathrm{G}$ broad band at $3154 \mathrm{~cm}^{-1}$ increased after the introduction of silver by UV irradiation.

SDS is characterised by several Raman modes localised at $600 \mathrm{~cm}^{-1}$, which is assigned to an $\mathrm{SO}_{3}$ vibration; $840 \mathrm{~cm}^{-1}$, which is assigned to $\mathrm{RO}-\mathrm{SO}_{3}$ stretching vibrations; $1087 \mathrm{~cm}^{-1}$, which is assigned to C-C stretching vibrations; and 1298, 1438, 2848 and $2881 \mathrm{~cm}^{-1}$, which are assigned to the $\mathrm{CH}_{2}$ vibration ${ }^{31}$. The fine profile of the SDS powder shows much more detail about the Raman vibrations of the SDS molecules (see Supplementary Information Figure
S4). Preliminary measurements of SDS deposited from a 1-g.L $\mathrm{L}^{-1}$ solution onto a silver SERS substrate, with average roughness equal to $22 \mathrm{~nm}$, do not show the SDS Raman modes (Figure 3a). The SDS modes are not visible in SDS@SWCNT samples in different chemical surroundings, except for a weak feature at $2850-2900 \mathrm{~cm}^{-1}$, which is assigned to the $-\mathrm{CH}_{2}$ vibrations of SDS in UV-Ag@SDS@SWCNT. The SERS measurement of the SDS solution also does not provide a significant Raman signal, possibly because the SDS molecules are non-resonant with the $514.5 \mathrm{~nm}$ laser excitation, and the Raman cross-section of the alkyl chains is too low to produce an inelastic scattering signal from the small number of molecules.

Interestingly, the introduction of silver NPs to the SDS@SWCNTs drastically modifies the Raman spectra, as shown in Figure 3a, in a manner that is strongly related to the reduction conditions. For the UV-Ag@SDS@SWCNT samples, an extremely strong mode is present at $969 \mathrm{~cm}^{-1}$ with an intensity similar to that of the SWCNT G-band. The position of this peak is reproducible for 25 measurement points with various relative intensities. This extremely strong mode is observed under 514.5-nm excitation at various powers (ranging from $10 \mu \mathrm{W}$ to $2 \mathrm{~mW}$ ) and is stable as the acquisition time changes or after the samples are aged for 3 months. With a smaller amplitude, this mode is also visible at an excitation wavelength of $632.8 \mathrm{~nm}$ (Supplementary Information Figure S5). An additional strong mode is observed at $1201 \mathrm{~cm}^{-1}$ for the UV-Ag@SDS@SWCNT samples. This mode is also stable for acquisition times ranging from $1 \mathrm{~s}$ to $10 \mathrm{~min}$ and after the samples are aged for 3 months. Its intensity varies independently of the intensity of the $969 \mathrm{~cm}^{-1}$ mode. Nevertheless, these two extremely strong modes are not observed for Chem-Ag@SDS@SWCNTs (Figure 3a) or for Pd@SDS@ SWCNTs $^{32}$ (also see Supplementary Information Figure S3) under the same conditions. A few other additional weak modes are present at $455,627,814$ and $1053 \mathrm{~cm}^{-1}$. None of these vibration modes are visible in the other samples, as shown in Figure 3. This observation indicates that interesting physical and/or chemical effects are related to the UV-Ag@SDS@SWCNTs, which are discussed later in this paper.

\section{Discussion}

The combination of the two effects in the Ag@SDS@SWCNT nanostructures lead to a large EF enhancement at the inter-particle 

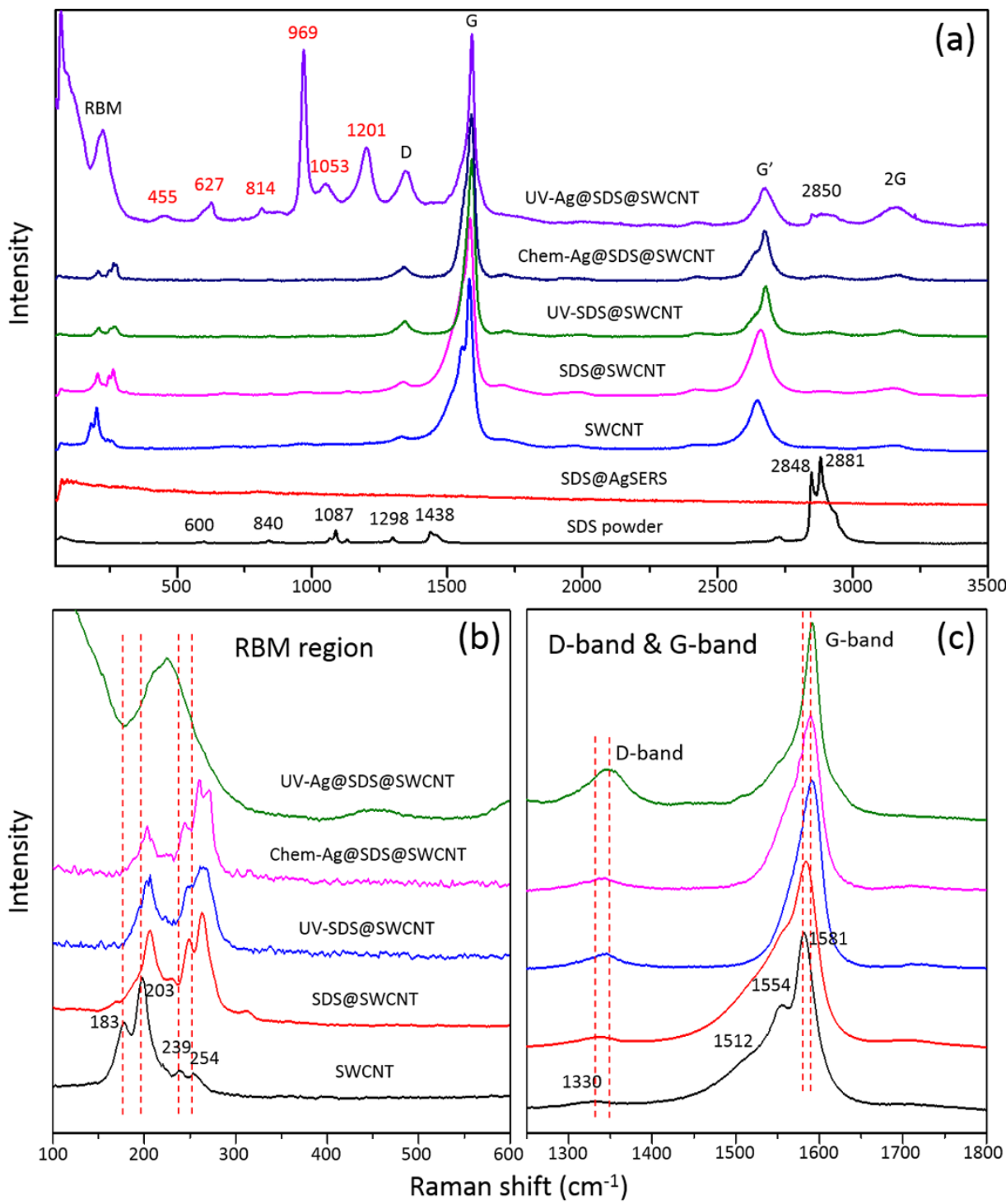

Figure 3 Raman spectra of SDS powder, SDS deposited on an Ag SERS substrate, pure SWCNTs, non-irradiated SDS-functionalised SWCNTs, UVirradiated SDS-functionalised SWCNTs, Ag nanoparticles precipitated onto SDS-functionalised SWCNTs by chemical reduction (Chem-Ag@SDS@ SWCNTs) and by photoreduction (UV-Ag@SDS@SWCNTs) (a). Comparison of the Raman spectra of the RBM mode (b), and the D-band \& G-band modes (c) of SWCNTs in different chemical surroundings.

junction $^{22,33}$; these effects combine the extremely large Raman crosssection of SWCNTs under resonant conditions, which is caused by the quantum confinement of the electronic states in this $1 \mathrm{D}$ mater$\mathrm{ial}^{34}$ and the strong field coupling between the NPs. Moreover, the self-organised molecular SDS structure in the gap shortens the distance between the silver NPs and the SWCNTs (approximately $1.5 \mathrm{~nm}$ ); because of this proximity, we can also expect a strong coupling of the LSPR of silver with the SWCNTs (see Figure 4a). However, according to previously reported calculations of the EF enhancement in the dimers and trimers of noble metal particles ${ }^{35}$, a strong increase in EF enhancement occurs when the inter-particle distance decreases to a few nanometers and when the incident polarisation is parallel to the chain axis ${ }^{22}$; for example, at 2-nm inter-particle distances, the EF enhancement has been estimated to be approximately $10^{5}$.

The strong coupling of LSPR was investigated by measuring the UV-visible absorption spectra of UV-Ag@SDS@SWCNT and Chem-Ag@SDS@SWCNT samples (see Figure 4b). The localised surface plasmon resonance (LSPR) of the silver NPs can be seen in the UV-visible spectrum at 410 nm for Chem-Ag@SDS@SWCNTs and at 404 nm for UV-Ag@SDS@SWCNTs. This blue shift could result from the smaller particle sizes and/or charge transfer to the NP surface. A few additional peaks are visible at lower energy for UVAg@SDS@SWCNTs (Figure 4c). Some of these peaks are assigned to the von Hove singularities of SWCNTs (see Supplementary Information Figure S6); other peaks centred at 465, 497, 537 and $620 \mathrm{~nm}$ have been previously assigned to coupled SPR modes in Ag dimers or Ag NP chains ${ }^{36-38}$ (see Supplementary Information Figure S7). Interestingly, no remarkable additional peaks are observed at lower energy for Chem-Ag@SDS@SWCNTs, which indicates that the coupling of LSPR is very different between the chemical reduction sample and the photoreduction sample.

We have extensively investigated the origin of the strongest additional mode at $969 \mathrm{~cm}^{-1}$ while considering the different interactions that occur in nanostructures. This mode's intensity is comparable to that of the G-band of carbon nanotubes. This mode does not appear in the SDS@SWCNT and Chem-Ag@SDS@SWCNT samples, and this mode is weak in the UV-SDS@Ag sample and very weak in Chem-SDS@Ag sample (see Figure 5). Furthermore, this mode is not present for the SDS molecules (see Supplementary Information Figure S4). The physics of the intermediate frequency modes (IFM) 

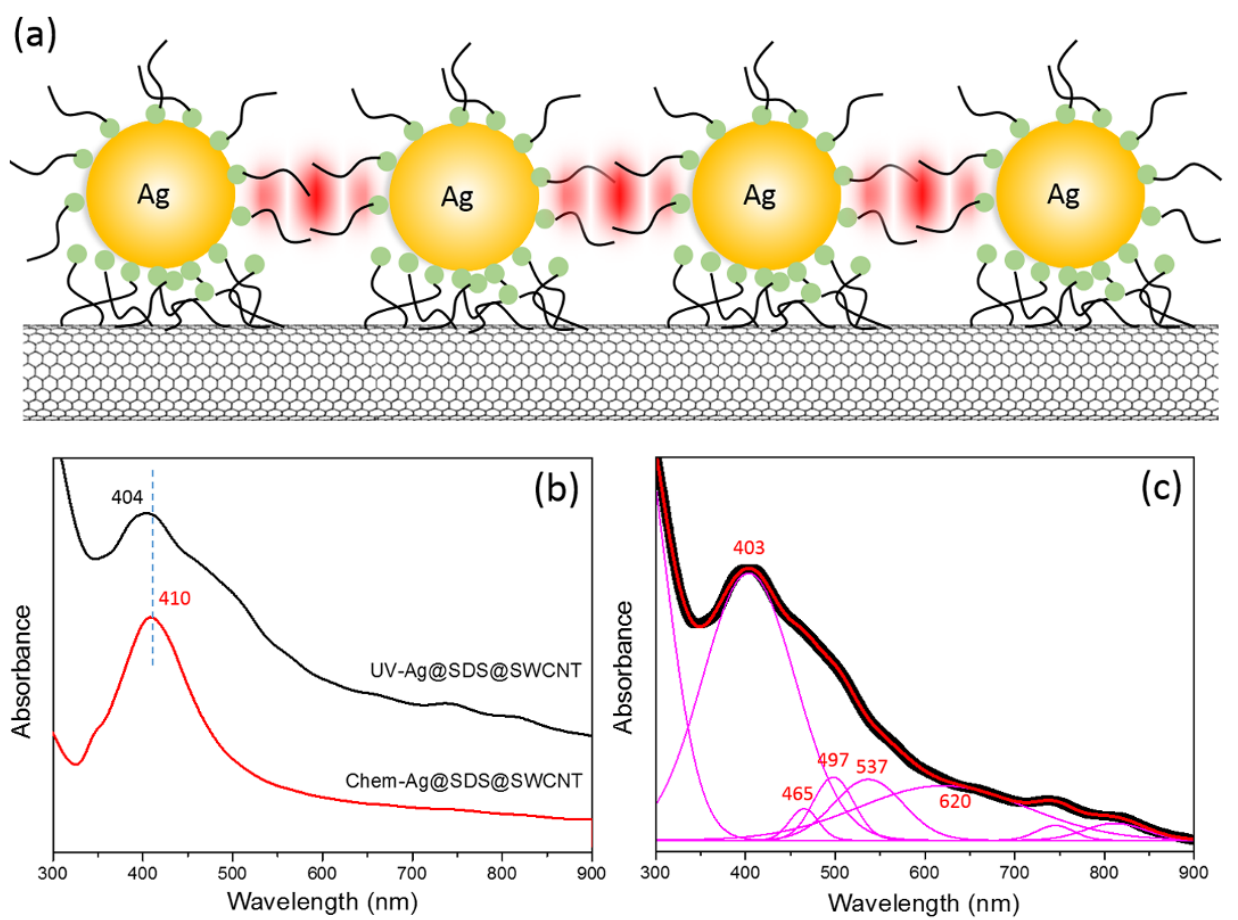

Figure 4 Schematic representation of LSPR coupling between Ag dimers (a), UV-visible absorption spectra of Ag nanoparticles precipitated onto SDS-functionalised SWCNTs by chemical reduction (Chem-Ag@SDS@SWCNT) and by photoreduction (UV-Ag@SDS@SWCNT) (b) and peak fitting of UV-Ag@SDS@SWCNTs (c). Black line and red line in (c) are original and simulated spectra, respectively.

of the SWCNT between $600-1100 \mathrm{~cm}^{-1}$ is complex, and only one of them, the intermediate frequency mode $($ IFM +$)$, appears at this position $\left(960 \mathrm{~cm}^{-1}\right)$, which corresponds to a two-phonon double resonance $^{39}$; however, this IFM+ mode is very weak compared to the strong $969 \mathrm{~cm}^{-1}$ mode found in our sample. Nevertheless, a mode at $974 \mathrm{~cm}^{-1}$ has previously been observed for SDS-functionalised silica ${ }^{40}$ and has been assigned to the $v_{\text {as }}(\mathrm{C}-\mathrm{O}-\mathrm{S})$ vibration. In their SERS experiments of $\mathrm{SO}_{2}$ adsorbed on silver, Matsuta et al. ${ }^{41}$ observed a mode at $925 \mathrm{~cm}^{-1}$ due to the $\mathrm{SO}_{3}{ }^{2-}$ group, but under an oxidative atmosphere $\left(\mathrm{SO}_{2} / \mathrm{O}_{2}\right.$ mixture), a new peak resulting from sulphur oxidation $\left(\mathrm{SO}_{4}{ }^{2-}\right)$ appeared at $960 \mathrm{~cm}^{-1}$. In our samples, the extremely strong mode at $969 \mathrm{~cm}^{-1}$ could result from a specific silver/sulphate group interaction that is induced by UV irradiation. The Raman shift position of this mode coincides with the strong Raman modes of silver sulphate, $\mathrm{Ag}_{2} \mathrm{SO}_{4}$, at $968 \mathrm{~cm}^{-1}$ (see Figure 5). Due to the hydrophilic silver/SDS interaction, the highly polarisable sulphate group is expected to behave as a nucleation site for silver photo-nucleation. After particle growth, SDS molecules remain at the surface of the silver NP, and the sulphate groups are mainly confined to a region where the EF is strong, i.e., in the gap between silver NP dimers and in the gap between the silver NPs and SWCNTs.

The extremely large intensity of this mode may also be due to the occurrence of a charge transfer mechanism at the surface of the silver particles. A schematic representation is given to describe the charge transfer effect for the enhanced Raman vibration of the $969 \mathrm{~cm}^{-1}$ mode (see Figure 6). The HOMO and LUMO levels of SDS are 7.66 and $2.88 \mathrm{eV}$, respectively, from the vacuum energy level ${ }^{42}$. The work function of $\mathrm{Ag}$ has been reported as $4.26 \mathrm{eV}^{43}$. The work function of SWCNTs is $4.5 \mathrm{eV}^{44}$, while the band gap $(0.6 \sim 0.8 \mathrm{eV})$ according to the theoretical value of semiconducting SWCNTs with diameters distributed at approximately $1.0 \sim 1.3 \mathrm{~nm}^{45}$, which is the diameter distribution of the SWCNTs used in our study. The energy difference is $1.38 \mathrm{eV}(\lambda=898 \mathrm{~nm})$ between the $\mathrm{E}_{\mathrm{F}}$ of $\mathrm{Ag}$ and the LUMO of SDS and $1.92 \sim 2.02 \mathrm{eV}(\lambda=646 \sim 614 \mathrm{~nm})$ between the HOMO of SWCNTs and the LUMO of SDS. Hence, both the

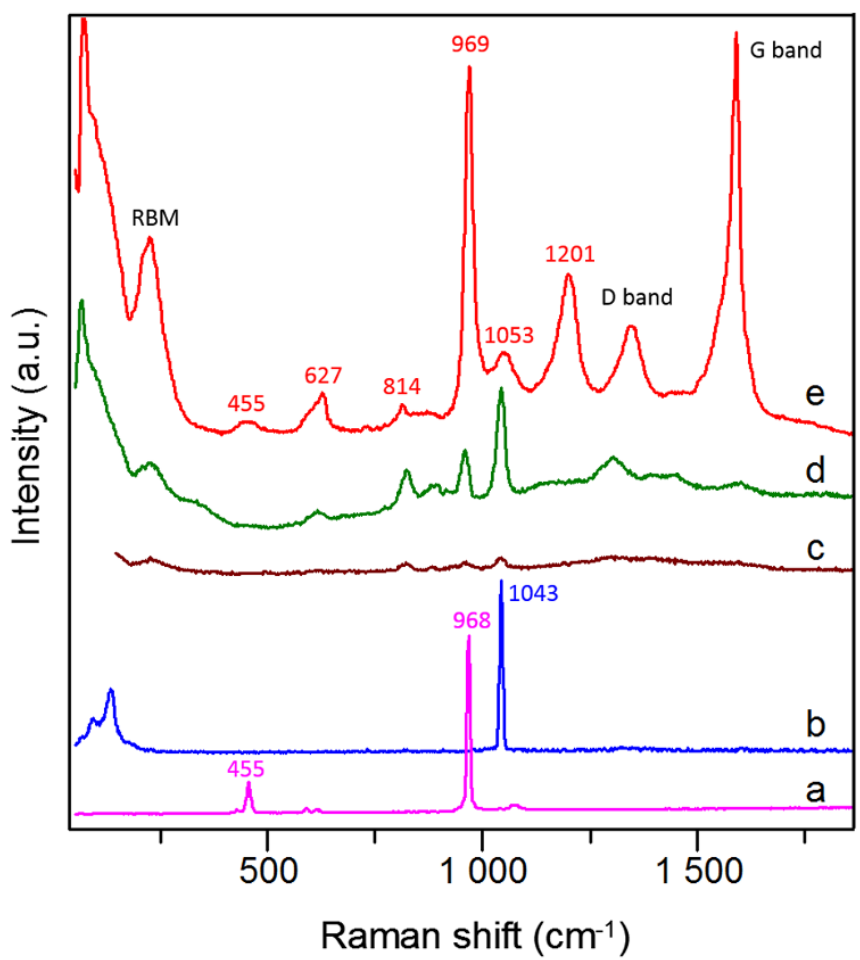

Figure $5 \mid$ Raman spectra of silver sulphate $\left(\mathrm{Ag}_{2} \mathrm{SO}_{4},(\mathrm{a})\right)$, silver nitrate $\left(\mathrm{AgNO}_{3},(\mathrm{~b})\right), \mathrm{Ag}$ nanoparticles obtained by the chemical reduction of the silver nitrate solution in the presence of SDS Chem-Ag@SDS (c), Ag obtained by the photoreduction of the silver nitrate solution in the presence of SDS UV-Ag@SDS (d) and Ag nanoparticles precipitated onto SDS-functionalised SWCNTs by photoreduction(UV-Ag@SDS@ SWCNTs) (e). 
514.5-nm laser and the 632.8-nm laser can provide sufficient energy for electron excitation and charge transfer from Ag to SDS. The 514.5-nm laser is also effective for the change transfer from the HOMO of SWCNTs to the LUMO of SDS. By contrast, the 632.8$\mathrm{nm}$ laser only excites the charge transfer of some SWCNTs when the energy gap is less than $\lambda_{\max }=632.8 \mathrm{~nm}$. The excited electrons enter the LUMO of SDS from the conduction band of the Ag nanoparticles and from the LUMO of the SWCNTs, which results in highly polarised $\mathrm{SO}_{4}{ }^{2-}$ groups and thus greatly enhances the Raman vibration at $969 \mathrm{~cm}^{-1}$. In this case, the strong differences in the Raman intensities observed for the Chem- and UV-samples are related not only to the particle size distributions (because the NPs obtained by chemical reduction are supposed to produce strong EF enhancement under 514.5-nm wavelength excitation) but also to surface interactions. According to the "adatom model", which predicts enhanced Raman scattering by adsorbate vibrations through the photonexcited charge transfer transition from localised electronic states at sites of atomic-scale roughness ${ }^{46,47}$ (see Supplementary Information Figure S8), the adsorbed sulphate group of the SDS surfactant could stabilise the Ag adatoms formed during the photoreduction process. By contrast, under chemical reduction, the nucleation sites are different and are related to the presence of a reducing agent in the solution. In the latter case, the stabilisation of adatoms by sulphate groups is not predominant. Electrochemical SERS measurements ${ }^{48}$ have suggested that the adatom-ligand surface complex is assumed to dissociate when the electrode potential is sufficiently large for the ligands to desorb. In our experiments, the number of SERS active sites could vary with the number of adatoms, unstable clusters and surface defects on silver NPs, which are expected to be very different between chemical reduction and photoreduction.
The second strongest Raman mode occurs at $1201 \mathrm{~cm}^{-1}$ in UVAg@SDS@SWCNTs. This mode is not visible on the Raman spectrum of SDS molecules, and no other Raman modes are expected in this region. However, the infrared spectra of carbohydrate $\mathrm{O}$ sulphates ${ }^{49}$ are characterised by a strong $v_{a s} S=O$ bond at $\sim 1230 \mathrm{~cm}^{-1}$. This IR mode is Raman non-active; nevertheless, an IR-active mode may not be ruled out for the SERS spectra because of the strong influence from the $\mathrm{EF}^{50}$. The selection rules can be altered by the change in symmetry due to the image charge in the metal ${ }^{51}$, by a change in site symmetry with surface bonding ${ }^{52}$ or by the nature of the EF. An internal EF has been shown to change the Raman selection rules in semiconductors that lack a symmetry centre ${ }^{53}$. When the gradient of the EF is large, such as at a metallic surface, the selection rules can also be modified ${ }^{54}$.

It is difficult to quantify the improvement in the Raman signal due to charge transfer; indeed, the extremely strong Raman mode observed at $969 \mathrm{~cm}^{-1}$ results from a charge transfer between the sulphate group, and silver cannot be observed in pure SDS. The $1201 \mathrm{~cm}^{-1}$ mode is not Raman active in SDS; hence, no reference value can be used to calculate the enhancement of the strongest modes. Both with and without enhancement, only the high frequency modes $\left(-\mathrm{CH}_{2}\right.$ at $\left.2850-2890 \mathrm{~cm}^{-1}\right)$ are observed. A rough estimation of this enhancement factor $(\mathrm{G})$ is the following:

$$
G=\frac{I_{\text {SERS }}}{V_{\text {SERS }}} \times \frac{V_{\text {ref }}}{I_{\text {ref }}}
$$

where reference spectrum is taken on SDS powder. The $V_{\text {SERS }}$ has been estimated to be approximately $2 \times 10^{4} \mathrm{~nm}^{3}$ and accounts for an average distance of $50 \mathrm{~nm}$ between the SWCNTs and a spot diameter
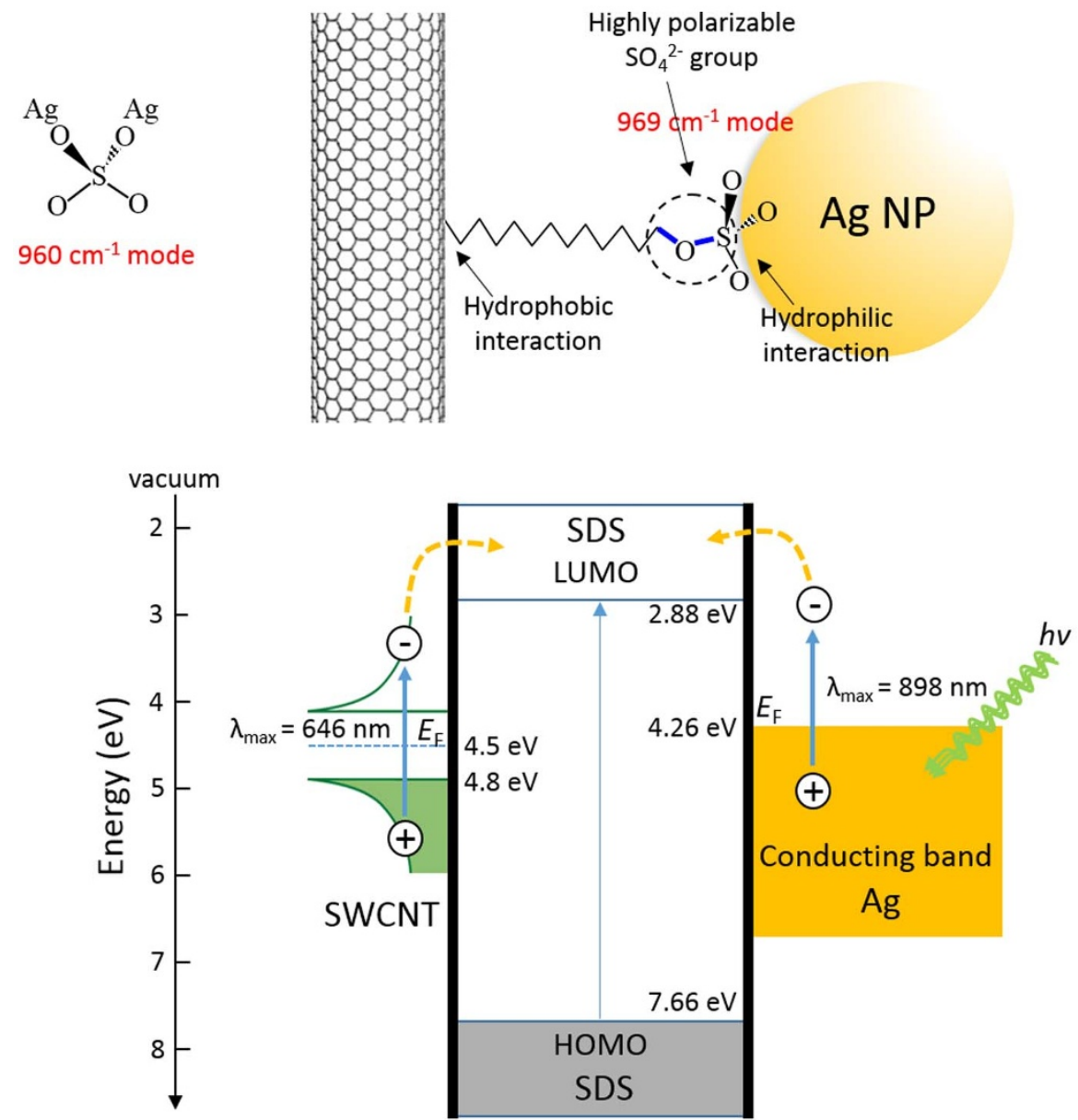

Figure $6 \mid$ Schematic representation of the enhanced Raman vibration of the $969 \mathrm{~cm}^{-1}$ mode. The band gap of the SWCNTs shown in the scheme is $0.6 \mathrm{eV}$ for a diameter of $\sim 1.3 \mathrm{~nm}$. 
of approximately $0.5 \mu \mathrm{m}$ (see Supplementary Information Figure S9). Due to the weak absorption of the SDS powder at $514.5 \mathrm{~nm}$, the $V_{r e f}$ is the confocal volume of approximately 0.5 to $1 \mu \mathrm{m}^{3}$. Under the same excitation power and time conditions, the intensity ratio $\frac{I_{S E R S}}{I_{\text {ref }}} \approx 0.1$. As a result, the enhancement factor is estimated to be $\sim 10^{4}-10^{5}$ for the high-frequency modes. Charge transfer supports a very large enhancement of the Raman signal.

Strong modifications are also observed for UV-Ag@SDS@ SWCNTs in the RBM spectral region, consisting of a new broad and strong mode at $226 \mathrm{~cm}^{-1}$. The large number of very small silver NPs that interact strongly with the SWCNTs via the SDS bridging molecules modifies the intertube forces. A calculation of the Raman intensity shows that the RBM of the single SWCNT disappears in bundles to give rise to two strong peaks due to the intertube forces that prevail over the elastic forces of the tube ${ }^{55}$. Another explanation for this $226 \mathrm{~cm}^{-1}$ mode could be the molecular oxygen species on the surface of silver, as suggested by Waterhouse et al. ${ }^{56}$

The strong modifications of the Raman spectra observed at the intermediate frequencies $\left(400-1250 \mathrm{~cm}^{-1}\right)$ are associated with modifications of the G-band. It has been previously suggested that the line shape of this mode is related to the splitting of $G$ modes and to the contribution of the BWF mode that results from the plasmon coupling between metallic tubes ${ }^{57}$. However, the BWF mode, which is considered to be a sensitive experimental probe of plasmon effects in SWCNT bundles, is not resonant under 514.5-nm excitation. Here, we suggest that the strong narrowing of the G-band in the UV-Ag@SDS@SWCNT samples could result from a selective enhancement of one of the Raman scattering modes induced by the Ag chain along the nanotube. The strong interaction between the SDS sulphate group and the silver NPs in the UV-irradiated samples and the related charge transfer could be responsible for the changes in the G-band ${ }^{58}$.

From these experiments, it is evident that UV exerts the strongest effects. Usually, very small Ag NPs are not expected to significantly enhance the field ${ }^{59,60}$. However, the calculations are difficult for these particle sizes because dielectric functions do not include nonlocal or size-dependant dielectric functions for particles whose diameter is close to the bulk mean electron free path, typically less than $5 \mathrm{~nm}$. The electron tunnelling between NPs along Ag NP chains with very small gaps could also move the LSPR to a higher frequency, but this mechanism is still under discussion and requires a quantum treatment.

This work demonstrated for the first time that the unique structure of very small Ag NPs (approximately 4-7 nm), which were created from a template of self-organised SDS molecules on SWCNTs, generated strong Raman scattering at $969 \mathrm{~cm}^{-1}$ and $1201 \mathrm{~cm}^{-1}$. The origin of these extremely strong modes is clearly related to the UV photoreduction process. We assigned the extremely strong mode at $969 \mathrm{~cm}^{-1}$ to the sulphate-silver interaction at the NP surface; this interaction is associated with a charge transfer mechanism that results from the UV photoreduction process. The $1201 \mathrm{~cm}^{-1}$ mode could be an IR-active mode of the SDS that is observed in our sample because of an alteration of the selection rules due to symmetry changes. The Raman intensities strongly depend on the reduction conditions. We suggest that not only particle sizes but also defects on the NP surface that during the nucleation mechanism could be responsible for this strong scattering. With UV reduction, the sulphate groups of the SDS surfactant act as a nucleation site, stabilising the silver complex on the NP surface. Most of the SERS experiments show that very small NPs do not produce a strong field enhancement; however, numerical simulations using Maxwell's equations lead to questionable results when the particle sizes become smaller than the mean electron free path or when the distance between the NPs in the Ag chains could lead to significant electron tunnelling. The "adatom model" proposed nearly twenty years ago could support our observations on the strong differences between the chemically reduced and UV photoreduced samples. The nucleation mechanisms are very different, and a silver-sulphate complex could be stabilised in the latter case, giving rise to a larger number of SERS sites. Moreover, we also demonstrated that the presence of SWCNTs is necessary to produce the strong Raman scattering. The mechanism is not yet clearly elucidated, but we demonstrated that the Raman modes of the SWCNTs (RBM and G-band) are also strongly modified by the presence of very small silver NPs along the nanotubes.

\section{Methods}

Preparation of SDS@SWCNTs. The arrangement of noble metal NPs, which are tailored by the supramolecular self-assembly of SDS molecules that are wrapped on HiPco SWCNTs, is obtained by chemical synthesis as previously described for palladium ${ }^{23}$. During the first step, $40 \mathrm{mg}$ of commercial HiPco SWCNTs (Carbon Nanotechnologies Inc., Houston, TX, USA) are dispersed in $80 \mathrm{~mL}$ of distilled water with $400 \mathrm{mg}$ of sodium dodecyl sulphate (SDS) (Kishida Chemical Co., LTD, Osaka, Japan). After grinding of the sample with 5-mm media zirconia balls during a 4-hour ultrasonication procedure, the resulting black dispersion was centrifuged twice $(8000 \mathrm{rpm}, 1 \mathrm{hr})$, yielding a well-dispersed supernatant of SWCNTs.

Preparation of Ag@SDS@SWCNT. In the present work, the reduction of silver salt at the surface of an SDS-functionalised SWCNT suspension was achieved by using photoreduction (UV-Ag@SDS@SWCNTs) and chemical reduction (Chem-Ag@ SDS@SWCNTs). In the photoreduction method, silver nanoparticles were obtained by the addition of $1 \mathrm{~mL}$ of an aqueous solution of silver nitrate $\left(\mathrm{AgNO}_{3}\right)(0.02 \mathrm{M})$ into $10 \mathrm{~mL}$ of the resulting SWCNT dispersion and then subjected to supersonic treatment. The $\mathrm{Ag}^{+}$ions were then reduced by photoreduction using a Spectroline hand-held UV lamp $\left(\lambda_{\max }=254 \mathrm{~nm}\right)$. In the chemical reduction method, the $\mathrm{Ag}^{+}$ ions were reduced by the addition of a fresh aqueous sodium borohydride $\left(\mathrm{NaBH}_{4}\right)$ solution $(0.01 \mathrm{M})$.

Preparation SDS@Ag and SDS on Ag SERS. For better interpretation of the results, further Raman experiments were also performed with reference samples, including SDS on Ag SERS substrates, SDS@Ag nanoparticles (obtained by chemical- and photoreduction methods),Pd@SDS@SWCNTs and UV-irradiated SDS@SWCNTs. The Raman spectra of SDS were obtained for three forms: SDS powder; SDS films evaporated on a glass substrate from an aqueous solution $\left(1 \mathrm{~g} . \mathrm{L}^{-1}\right)$; and SDS films evaporated on silver SERS substrates (showing an average roughness of approximately $22 \mathrm{~nm}$ ).

Characterisation of samples. After silver reduction, the nanostructures were deposited on a carbon-coated $\mathrm{Cu}$ grid and studied by transmission electron microscopy (JEM-2100F, JEOL, Japan). The absorption spectra were measured using a transmission UV-visible spectrophotometer (UV-3100PC, Shimadzu, Japan). Infrared spectra were obtained by diffuse reflectance FTIR (IR Prestige-21 spectrometer, Shimadzu, Japan). For the Raman experiments, $10 \mu \mathrm{L}$ drops of the SWCNT suspensions were deposited onto glass slides and dried in air at room temperature. We used a Labram HR800 Horiba Jobin-Yvon spectrometer with an $\mathrm{Ar}^{+}$laser beam excitation at $514.5 \mathrm{~nm}$. The beam, whose power ranged from $10 \mu \mathrm{W}$ to $2 \mathrm{~mW}$ to assess thermal effects, was focussed on the samples with a $100 \times$ objective (NA 0.9), leading to an illuminated area of approximately $0.7 \mu \mathrm{m}^{2}$, which includes several SWCNT bundles. For additional Raman experiments, a HeNe 632.8-nm laser excitation was used.

1. Bui, M. P. N. et al. Electrocatalytic reduction of hydrogen peroxide by silver particles patterned on single-walled carbon nanotubes. Sens. Actuators B 150, 436-441 (2010).

2. Sanles-Sobrido, M. et al. Label-free SERS detection of relevant bioanalytes on silver-coated carbon nanotubes: the case of cocaine. Nanoscale 1, 153-158 (2009).

3. Wang, T. et al. Silver nanoparticle-decorated carbon nanotubes as bifunctional gas-diffusion electrodes for zinc-air batteries. J. Power Sources 195(13), 4350-4355 (2010).

4. Wee, G. et al. Particle size effect of silver nanoparticles decorated single walled carbon nanotube electrode for supercapacitors. J. Electrochem. Soc. 157(2), A179-A184 (2010).

5. Chen, Z. et al. Ag nanoparticles coated SWCNT with surface enhanced Raman scattering (SERS) signals. J. Nanosc. Nanotech. 10, 8538-8543 (2010).

6. Sahoo, S., Husale, S., Karna, S., Nayak, S. K. \& Ajayan, P. M. Controlled assembly of Ag nanoparticles and carbon nanotube hybrid structures for biosensing. J. Am. Chem. Soc. 133, 4005-4009 (2011).

7. Chen, Y. C., Young, R. J., Macphersonb, J. V. \& Wilson, N. R. Silver-decorated carbon nanotube networks as SERS substrates. J. Raman Spectrosc. 42, 1255-1262 (2011).

8. Becker, M. et al. The SERS and TERS effects obtained by gold droplets on top of Si nanowires. Nano Lett. 7, 75-80 (2007).

9. Eckel, R. et al. Fluorescence-emission control of single CdSe nanocrystals using gold-modified AFM tips. Small 3, 44-49 (2007). 
10. Kim, Z. H. \& Leone, S. R. High-resolution apertureless near-field optical imaging using gold nanosphere probes. J. Phys. Chem. B 110, 19804-19809 (2006).

11. Anderson, N., Hartschuh, A., Cronin, S. \& Novotny, L. Nanoscale vibrational analysis of single-walled carbon nanotubes. J. Am. Chem. Soc. 127, 2533-2537 (2005).

12. Gucciardi, P. G. \& Valmalette, J. C. Different longitudinal optical-transverse optical mode amplification in tip enhanced Raman spectroscopy of GaAs(001) Appl. Phys. Lett. 97, 263104 (2010).

13. Merlen, A. et al. Depolarisation effects in tip-enhanced Raman spectroscopy J. Raman Spectrosc. 40, 1361-1370 (2009).

14. Huber, A. J., Ziegler, A., Kock, T. \& Hillenbrand, R. Infrared nanoscopy of strained semiconductors. Nature Nanotech. 4, 153-157 (2009).

15. Stiegler, J. M. et al. Nanoscale free carrier profiling on individual semiconductor nanowires by infrared near-field nanoscopy. Nano Lett. 10, 1387-1392 (2010).

16. Tseng, C. H. \& Chen, C. Y. Growth of Ag nanoparticles using plasma-modified multi-walled carbon nanotubes. Nanotechnology 19, 035606 (2008).

17. Lin, Y. et al. Rapid, solventless, bulk preparation of metal nanoparticle-decorated carbon nanotubes. ACS Nano 3, 871-884 (2009).

18. Liz-Marzan, L. M. Tailoring surface plasmons through the morphology and assembly of metal nanoparticles. Langmuir 22, 32-41 (2006).

19. Yang, Y., Shi, J. L., Tanaka, T. \& Nogami, M. Self-assembled silver nanochains for surfaceenhanced Raman scattering. Langmuir 23, 12042-12047 (2007).

20. Bouhelier, A. et al. Electromagnetic interactions in plasmonic nanoparticle arrays. J. Phys. Chem. B 109, 3195-3198 (2005)

21. Hao, E. \& Schatz, G. C. Electromagnetic fields around silver nanoparticles and dimers. J. Chem. Phys. 120, 357-366 (2004).

22. García-Vidal, F. J., Pitarke, J. M. \& Pendry, J. B. Silver-filled carbon nanotubes used as spectroscopic enhancers. Phys. Rev. B 58, 6783-6786 (1998).

23. Tan, Z., Abe, H., Naito, M. \& Ohara, S. Arrangement of palladium nanoparticles templated by supramolecular self-assembly of SDS wrapped on single-walled carbon nanotubes. Chem. Commun. 46, 4363-4365 (2010).

24. Tan, Z., Xu, H., Abe, H., Naito, M. \& Ohara, S. Anisotropic polyhedral selfassembly of Ag-CNT nanocomposites. J. Nanosci. Nanotech. 10, 3978-3982 (2010).

25. O'Connell, M. J. et al. Band gap fluorescence from individual single-walled carbon nanotubes. Science 297, 593-596 (2002)

26. Richard, C., Balavoine, F., Schultz, P., Ebbesen, T. W. \& Mioskowski, C. Supramolecular self-assembly of lipid derivatives on carbon nanotubes. Science 300, 775-778 (2003).

27. Cai, Z. et al. One-pot synthesis of silver particle aggregation as highly active SERS substrate. J. Raman Spectrosc. 42, 5-11 (2011).

28. Dillon, A. C., Yudasaka, M. \& Dresselhaus, M. S. Employing Raman spectroscopy to qualitatively evaluate the purity of carbon single-wall nanotube materials. J. Nanosci. Nanotech. 4, 691-703 (2004).

29. de la Chapelle, M. L. et al. Raman studies on single Walled carbon nanotubes produced by the electric arc technique. Carbon 36, 705-708 (1998).

30. de Frein, C., Lestini, E., Quirke, N. \& Zerulla, D. Environmental effects on the Raman spectra of single walled carbon nanotubes. Phys. Status Solidi B 250, 2635-2638 (2013).

31. Picquart, M. Vibrational model behavior of SDS aqueous solutions studied by Raman scattering. J. Phys. Chem. 90, 243-250 (1986).

32. Tan, Z., Abe, H. \& Ohara, S. Ordered deposition of Pd nanoparticles on sodium dodecyl sulfate-functionalised single-walled carbon nanotube. J. Mater. Chem. 21, 12008-12014 (2011).

33. Wang, Z. B. et al. The influences of particle number on hot spots in strongly coupled metal nanoparticles chain. J. Chem. Phys. 128, 094705 (2008).

34. Jorio, A. et al. Joint density of electronic states for one isolated single-wall carbon nanotube studied by resonant Raman scattering. Phys. Rev. B 63, 245416 (2001).

35. Jain, P. K. \& El-Sayed, M. A. Surface plasmon coupling and its universal size scaling in metal nanostructures of complex geometry: elongated particle pairs and nanosphere trimers. J. Phys. Chem. C 112, 4954-4960 (2008).

36. Nordlander, P., Oubre, C., Prodan, E., Li, K. \& Stockman, M. I. Plasmon hybridization in nanoparticle dimers. Nano Lett. 4, 899-903 (2004).

37. Pérez-González, O., Zabala, N. \& Aizpurua, J. Optical characterization of charge transfer and bonding dimer plasmons in linked interparticle gaps. New J. Phys. 13, 083013 (2011).

38. Kim, D. S. et al. Real-space mapping of the strongly coupled plasmons of nanoparticle dimers. Nano Lett. 9, 3619-3625 (2009).

39. Dresselhaus, M. S., Dresselhaus, G., Saito, R. \& Jorio, A. Raman spectroscopy of carbon nanotubes. Phys. Rep. 409, 47-99 (2005).

40. Wang, W., Gu, B. \& Liang, L. Effect of anionic surfactants on synthesis and selfassembling of silica colloidal nanoparticles. J. Colloid Interface Sci. 313, 169-173 (2007)

41. Matsuta, H. \& Hirokawa, K. SERS observation of the adsorption behavior of $\mathrm{SO}_{2}$ on silver powder surfaces at nearly real environmental conditions. Appl. Surf. Sci. 27, 482-486 (1987)
42. Basheer, M. M., Perles, C. E., Volpe, P. L. O. \& Airoldi, C. Theoretical investigations of the effect of a homologous series of anionic surfactants on the metabolism bioactivity. J. Solution Chem. 35, 625-637 (2006).

43. Michaelson, H. B. The work function of the elements and its periodicity. J. Appl. Phys. 48, 4729-4733 (1977).

44. Kymakis, E., Alexandrou, I. \& Amaratunga, G. A. J. High open-circuit voltage photovoltaic devices from carbon-nanotubes-polymer composites. J. Appl. Phys. 93, 1764-1768 (2003).

45. Kataura, H. et al. Optical properties of single-wall carbon nanotubes. Synth Metals 103, 2555-2558 (1999).

46. Billmann, J., Kovacs, G. \& Otto, A. Enhanced Raman effect from cyanide adsorbed on a silver electrode. Surf. Sci. 92, 153-173 (1980)

47. Otto, A. Surface enhanced Raman scattering (SERS), what do we know? Appl. Surf. Sci. 6, 309-355 (1980).

48. Otto, A., Billmann, J., Eickmans, J., Erturk, U. \& Penenkofer, C. The "adatom model" of SERS (surface enhanced Raman scattering): the present status. Surf. Sci. 138, 319-338 (1984).

49. Cabassi, F., Casu, B. \& Perlin, S. A. Infrared absorption and Raman scattering of sulfate groups of heparin and related glycosaminoglycans in aqueous solution. Carbohydr. Res. 63, 1-11 (1978).

50. Ayars, E. J., Hallen, H. D. \& Jahncke, C. L. Electric field gradient effects in Raman spectroscopy. Phys. Rev. Lett. 85, 4180-4183 (2000).

51. Moskovits, M. Surface selection rules. J. Chem. Phys. 77, 4408-4416 (1982)

52. Lippitsch, M. E. Ground-state charge transfer as a mechanism for surfaceenhanced Raman scattering. Phys. Rev. B 29, 3101-3110 (1984).

53. Schäffler, F. \& Abstreiter, G. Electric-field-induced Raman scattering: resonance, temperature, and screening effects. Phys. Rev. B 34, 4017-4025 (1986).

54. Sass, J. K., Neff, H., Moskovits, M. \& Holloway, S. Electric field gradient effect on the spectroscopy of adsorbed molecules. J. Phys. Chem. 85, 621-623 (1981)

55. Popov, V. N. \& Henrard, L. Evidence for the existence of two breathinglike phonon modes in infinite bundles of single-walled carbon nanotubes. Phys. Rev. B 63, 233407 (2001).

56. Waterhouse, G. I. N., Bowmaker, G. A. \& Metson, J. B. Oxygen chemisorption on an electrolytic silver catalyst: a combined TPD and Raman spectroscopic study. Appl. Surf. Sci. 214, 36-51 (2003).

57. Jiang, C. et al. Strong enhancement of the Breit-Wigner-Fano Raman line in carbon nanotube bundles caused by plasmon band formation. Phys. Rev. B 66 161404(R) (2002)

58. Corio, P. et al. Potential dependent surface Raman spectroscopy of carbon nanotubes. Chem. Phys. Lett. 370, 675-682 (2003).

59. Pustovit, V. N. \& Shahbazyan, T. V. SERS from molecules adsorbed on small Ag nanoparticles: A microscopic model. Chem. Phys. Lett. 420, 469-473 (2006).

60. Stamplecoskie, K. G. \& Scaiano, J. C. Optimal size of silver nanoparticles for surface-enhanced Raman spectroscopy. J. Phys. Chem. C 115, 1403-1409 (2011)

\section{Acknowledgments}

The authors acknowledge P.G. Gucciardi from CNR Messine and Marc Lamy de la Chapelle from Université Paris 13 for fruitful discussions.

\section{Author contributions}

J.V. and Z.T. contributed equally to this work. J.V. performed the Raman measurements, analysed the data, and wrote the main manuscript text. Z.T. synthesised the samples, performed the TEM and UV-Vis measurements, analysed the data, and contributed to the manuscript writing. H.A. and S.O. offered helpful discussion in the study. All authors reviewed the manuscript.

\section{Additional information}

Supplementary information accompanies this paper at http://www.nature.com/ scientificreports

Reprints and permission information is available online at http://npg.nature.com/ reprintsandpermissions

Competing financial interests: The authors declare no competing financial interests.

How to cite this article: Valmalette, J.-C., Tan, Z.Q., Abe, H. \& Ohara, S. Raman scattering of linear chains of strongly coupled Ag nanoparticles on SWCNTs. Sci. Rep. 4, 5238; DOI:10.1038/srep05238 (2014)

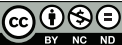

This work is licensed under a Creative Commons Attribution-NonCommercialNoDerivs 3.0 Unported License. The images in this article are included in the article's Creative Commons license, unless indicated otherwise in the image credit; if the image is not included under the Creative Commons license, users will need to obtain permission from the license holder in order to reproduce the image. To view a copy of this license, visit http://creativecommons.org/licenses/by-nc-nd/3.0/ 